LBNL-48996

To be published in

IEEE Transactions on Plasma Science

\title{
Two-dimensional Sample Temperature Modeling in Separation by Plasma Implantation of Oxygen (SPIMOX) Process
}

\author{
Tian, X. ${ }^{1}$; Chu, P.K. ${ }^{1}$; Anders, A. ${ }^{2}$ \\ ${ }^{1)}$ Department of Physics \& Materials Science, City University of Hong Kong, \\ 83 Tat Chee Avenue, Kowloon, Hong Kong, China \\ ${ }^{2)}$ Lawrence Berkeley National Laboratory, University of California, Berkeley, CA 94720, USA
}

July 25, 2001

This work was supported by the Director, Office of Science, U.S. Department of Energy under Contract No. DE-AC03-76SF00098 and Hong Kong Research Grants Council (CERG \# 9040412 or CityU 1003/99E and \#9040577 or CityU 1013/01E) as well as City University of Hong Kong (SRG \# 7001028). 


\title{
Two-dimensional Sample Temperature Modeling in
}

\section{Separation by Plasma Implantation of Oxygen (SPIMOX) Process}

\author{
Xiubo Tian (Member), Paul K. Chu (Senior Member)* \\ Department of Physics \& Materials Science, City University of Hong Kong, \\ 83 Tat Chee Avenue, Kowloon, Hong Kong, China \\ André Anders (Fellow) \\ Lawrence Berkeley National Laboratory \\ University of California, Berkeley, California 94720, USA
}

\begin{abstract}
Plasma immersion ion implantation (PIII) offers high throughput and efficiency in the synthesis of silicon-on-insulator (SOI) materials. In the separation by plasma implantation of oxygen (SPIMOX) process, the spatial and time variation of the sample temperature must be known and well controlled to ensure uniform buried oxide and silicon overlying layer thicknesses over the entire silicon wafer. In this paper, we describe a two-dimensional model and derive the temperature distribution on the silicon wafer with respect to time and other process parameters. Our results show laterally non-uniform heating by the incoming ions and the local temperature is influenced more by the sample voltage and thermal irradiation coefficient of the target than the pulse duration and plasma density. The model provides a simple and quick means to determine whether external heating will be needed to maintain the sample temperature at $600^{\circ} \mathrm{C}$ during the SPIMOX process.

* Corresponding author:

Prof. Paul K Chu

Department of Physics and Materials Science

City University of Hong Kong

83 Tat Chee Avenue

Kowloon, Hong Kong

Tel: $+852-27887724$

Fax: $+852-27889549$ or $+852-27887830$

Electronic mail: paul.chu@ cityu.edu.hk
\end{abstract}




\section{INTRODUCTION}

Silicon-on-insulator (SOI) is the preferred substrate for low-power, high-speed microelectronic devices, but suffers from high materials cost compared to bulk silicon [1]. In both the separation by implantation of oxygen (SIMOX) and Smart-Cut ${ }^{\circledR}$ processes, the high

manufacturing cost stems from the long time needed to implant a sufficiently high dose of oxygen or hydrogen, and the throughput is lower for larger wafers. Because the implantation time is independent of the wafer size, plasma immersion ion implantation (PIII) is a more efficient approach than beam-line ion implantation with regard to SOI formation [2-9]. The high ion dose rate and small equipment footprint are additional advantages.

In either the SIMOX or separation by plasma implantation of oxygen (SPIMOX) process, the sample temperature during implantation must be kept at $600^{\circ} \mathrm{C}$ [1]. In the SPIMOX process, since the ion dose uniformity depends on the process conditions and sample chuck geometry, the heat input due to incoming ions may not be uniform over the entire wafer and if the variation is large, local defects as well as non-uniform buried oxide and silicon overlayer thicknesses may result. This temperature variation can in fact impact the outcome of nonsemiconductor PIII applications [10].

Even though direct temperature monitoring is highly desirable in PIII, the high sample voltage and electrical / magnetic interference render common temperature measurement techniques impractical. It is even more difficult to measure in real time the temperature on multiple areas of the wafer. It is thus important to develop a model to map the temperature gradient over the entire sample. Blanchard developed a simple, time-dependent, lumped capacity thermal model [11], but unfortunately, it cannot provide multi-dimensional 
temperature information. In order to simulate the target temperature more accurately, a twodimensional model combining a plasma fluid model with the non-steady-state heat transfer formalism has been proposed [12]. In this work, we apply the model to investigate the SPIMOX process.

\section{SIMULATION}

The hardware parameters are based on the semiconductor PIII machine operating at the City University of Hong Kong and the instrument has been described in details elsewhere [13]. In our simulation, a $100 \mathrm{~mm}$ silicon wafer is laid on top of a $152 \mathrm{~mm}$ diameter, $52 \mathrm{~mm}$ thick stainless steel sample platen, and the high voltage feedthrough is shielded by a quartz tube.

Our model takes into account ions implanted into all areas including the silicon wafer and exposed sample platen (brim, side, and bottom).

The incident ion flux is derived by simulating the temporal sheath expansion using a twodimensional fluid model [14-16]. The target is immersed in oxygen plasma with density $n_{0}$ that is assumed to be spatially uniform before each voltage pulse. The evolution of the ion density $n_{i}$, ion velocity $v_{i}$, and potential $\phi$ is modeled using cold, collissionless fluid ions, Boltzmann electrons and Poisson's equation. In cylindrical coordinates, the two-dimensional equations of ion continuity and motion, Poisson's equation and Boltzmann relationship are:

$$
\begin{gathered}
\frac{\partial n}{\partial \tau}+\frac{1}{R} \frac{\partial}{\partial R}\left(R n u_{R}\right)+\frac{\partial}{\partial Z}\left(n u_{z}\right)=0 \\
\frac{\partial u_{R}}{\partial \tau}+u_{R} \frac{\partial u_{R}}{\partial R}+u_{z} \frac{\partial u_{R}}{\partial Z}=\frac{1}{2} \frac{\partial \varphi}{\partial R} \\
\frac{\partial u_{z}}{\partial \tau}+u_{R} \frac{\partial u_{z}}{\partial R}+u_{z} \frac{\partial u_{z}}{\partial Z}=\frac{1}{2} \frac{\partial \varphi}{\partial Z}
\end{gathered}
$$




$$
\frac{\partial^{2} \varphi}{\partial R^{2}}+\frac{1}{R} \frac{\partial \varphi}{\partial R}+u_{z} \frac{\partial^{2} \varphi}{\partial Z^{2}}=2\left[n-\exp \left(\frac{e \phi_{t}}{k T_{e}} \varphi\right)\right]
$$

The normalized dimensionless variables are $R=x / S_{0}, Z=z / S_{0}, \varphi=\phi / \phi_{t}, n=n_{i} / n_{0}, u_{R}=v_{i R} / v_{\max }$, $u_{Z}=v_{i Z} / v_{\max }, \tau=t / \omega_{p i}$, where $S_{0}=\left(-2 \varepsilon_{0} \phi_{t} / e n_{0}\right)^{0.5}$ is the planar ion-matrix sheath width, $v_{\max }=(-$ $\left.2 e \phi_{t} / m\right)^{0.5}$ is the velocity that an ion would gain if it fell through a potential drop $\phi_{t}$, and $\omega_{p i}=$ $\left(n_{0} e^{2} / m \varepsilon_{0}\right)^{0.5}$ is the ion plasma frequency. The equations are solved by the finite difference method. The simulation parameters are chosen based on actual experimental parameters: $n_{0}=$ $5.0 \times 10^{15}$ or $1 \times 10^{16} \mathrm{~m}^{-3}, \phi_{t}=-20$ or $-40 \mathrm{kV}, k T_{e}=4 \mathrm{eV}$, and an oxygen plasma $\left(\mathrm{O}_{2}{ }^{+}\right)$. The spatial distribution of the deposited energy derived from the ion flux for each pulse at two accelerating voltages is shown in Fig. 1. Due to the symmetry of the sample stage, only a quarter of the sample platen (right half of the top and upper half of the side) is shown. It can be observed that heating from ions impacting the side of the sample platen is quite substantial.

Owing to the relatively small ion projected range compared to the substrate thickness, the process can be modeled as a semi-infinite, one-dimensional solid with a uniform surface heat flux. In order to obtain an upper bound for a single pulse, the conduction and radiation terms can be eliminated, and the equation becomes [11]: $T(0, t)=\frac{2 q}{k} \sqrt{\frac{\kappa t}{\pi}}$, where $\kappa$ is the thermal diffusivity, $\rho=8020 \mathrm{~kg} / \mathrm{m}^{3}, \quad c=460 \mathrm{~J} / \mathrm{kgK}$, and $k=30 \mathrm{~W} / \mathrm{mK}$ for stainless steel. For simplicity, we assume that heat conductance through the silicon wafer (from top to bottom) is instantaneous compared to that through the stainless steel platen. In this case, a single $18 \mu \mathrm{s}$ pulse contributes a temperature rise of $0.76 \mathrm{~K}$. This is in line with the results (about $0.7 \mathrm{~K}$ ) measued by Chun, et al [17] thereby confirming the validity of our model. As this value is relatively small, the target temperature can be modeled by averaging the input power over the 
full cycle. The target temperature is simulated by solving the non-steady-state heat transfer equation by the finite difference method. In cylindrical coordinates, the equation is:

$$
k\left[\frac{1}{r} \frac{\partial}{\partial r}\left(r \frac{\partial T}{\partial r}\right)+\frac{\partial^{2} T}{\partial z^{2}}\right]+H=\rho c \frac{\partial T}{\partial T}
$$

where $\rho$ is the density of the target materials, $c$ is the heat capacity, $k$ is the thermal conductivity, and $H$ is the heating and cooling power corresponding to ion bombardment and thermal radiation. The energy deposited by the implanted ions is used as the input paramter. Heat loss by conduction via the high voltage feedthrough can be ignored on account of the thin stainless steel supporting rod. Therefore, the primary heat loss mechanism is radiation, and the thermal emissivity is assumed to be $\varepsilon=0.5$. The vacuum chamber wall is watercooled at $22^{\circ} \mathrm{C}$, and we also assume that the target is initially at a temperature of $22^{\circ} \mathrm{C}$ or $295 \mathrm{~K}$.

\section{RESULTS and DISCUSSION}

The temperature distributions along the top (radial direction) and side (vertical direction) of the sample platen after 1, 300, and 1800 seconds implantation time are depicted in Fig. 2. The oxygen implantation conditions are: voltage $=-40 \mathrm{kV}$, pulse width $=18 \mu \mathrm{s}$, plasma density $=5 \times 10^{15} \mathrm{~m}^{-3}$, and pulsing frequency $=300 \mathrm{~Hz}$. It can be observed that the temperature near the side of the target (large radial and vertical distances) is higher than that near the center. It is interesting that there are three higher-temperature sites at the initial time stage (Fig. 2a) located at and near the sample edge. The temperature difference is not small across the entire target holder, but the radial variation along the top surface is only a few degrees within the silicon wafer range $(100 \mathrm{~mm})$ although the heat input is more nonuniform. The target temperature rises rapidly initially but more slowly later due to higher radiation 
heat loss at higher temperature. Based on the simulation results, the highest temperature induced by the incident energetic ions is $685 \mathrm{~K}$ under these conditions, and so the implantation power must be increased in order to heat the sample to $873 \mathrm{~K}\left(600^{\circ} \mathrm{C}\right)$ in the absence of external heating. Fig. 3 compares the influence of processing parameters on the equilibrium temperature at a pulsing frequency of $500 \mathrm{~Hz}$ without external heating or cooling. The results indicate that a variation of the implantation voltage has a larger effect on the target temperature than that of the plasma density or pulse duration. This trend is consistent with our experimental results obtained for different targets and processing parameters [18]. Fig. 4 shows the effect of the pulsing frequency on the equilibrium target temperature for a voltage of $40 \mathrm{kV}$, plasma density of $5 \times 10^{15} \mathrm{~m}^{-3}$, and pulse width of $18 \mu \mathrm{s}$. The rate of the temperature-rise gradually decreases with increasing pulsing frequency. The results indicate that it is not easy to reach $600^{\circ} \mathrm{C}$ using ion implantation alone. For instance, for $\varepsilon=0.5$, a high frequency of $800 \mathrm{~Hz}$ is needed as shown in Fig. 4. If such a high power is not achievable due to hardware limitation, external heating must be used. The ability to calculate the ion heating power based on a chosen set of processing conditions is thus important to the process engineers in order to determine whether external heating is necessary. In addition, the temperature distribution over the entire silicon wafer derived from the model can be combined with an empirically determined temperature at one spot on the wafer using a pyrometer or thermocouple to enhance the accuracy. Fig. 5 also illustrates the effects of different radiation heat losses $(\varepsilon=0.5$ and 0.1$)$ and the needed heating power. The influence is quite significant and the heat emissivity of the sample chuck surface should also be considered when designing the PIII conditions. A silicon-compatible coating with low heat emissivity may reduce heat loss enough to allow for the use of a lower frequency or power or simply require some form of non-contact radiation heating. 


\section{CONCLUSION}

A simple model is used to predict and explain the sample temperature distribution during oxygen PIII in the SPIMOX process. The method employs a two-dimensional fluid model and temperature governing equation to describe the temperature rise and variation on the sample. The results show that ion heating is spatially non-uniform even though the temperature gradient across the sample is not substantial. A variation of the implantation voltage has a larger influence on the target temperature than that of the plasma density or pulse duration. The model can be used to determine whether external heating is necessary for different processing conditions. The results also indicate that heat loss due to radiation is very significant in the SPIMOX process and a suitable coating materials on the sample chuck may obviate the need for an external heating source. Last but not least, the model can be combined with the temperature measured experimentally oin one spot of the wafer to more accurately determine the lateral temperature distribution. The model can be easily applied to other PIII processes, e.g. water or hydrogen PIII by simply replacing oxygen with the appropriate ion species.

\section{Acknowledgments}

This work described in this paper was jointly supported by grants from the Hong Kong Research Grants Council (CERG \# 9040412 or CityU 1003/99E and \# 9040577 or CityU 1013/01E) as well as City University of Hong Kong (SRG \# 7001028). 


\section{References}

1 S. S. K. Iyer, X. Lu, J. B. Liu, J. Min, Z. N. Fan, P. K. Chu, C. M. Hu, and N. W. Cheung, "Separation by Plasma Implantation of Oxygen (SPIMOX) Operational Phase Space”, IEEE Trans. Plasma Sci. vol. 25, pp. 1128-1135, 1997.

2 J. R. Conrad, J. L. Radtke, R. A. Dodd, F. J. Worzala and N. C. Tran, "Plasma source ionimplantation technique for surface modification of materials", J. Appl. Phys., vol. 62, pp. 4591-4596, 1987.

3 J. Tendys, I. J. Donnelly, M. J. Kenny and J. T. A. Pollock, "Plasma immersion ion implantation using plasmas generated by radio frequency techniques", Appl. Phys.Lett., vol. 53, pp. 2143-45, 1988.

4 B. Mizuno, I. Nakayama, N. Aoi, M. Kubota, and T. Komeda, "New doping method for subhalf micron trench sidewalls by using an electron cyclotron resonance plasma”, Appl. Phys.Lett., vol. 53, pp. 2059-61, 1988.

5 N. W. Cheung, "Plasma Immersion Ion Implantation for ULSI Processing", Nucl. Instr. Meth. B, vol. 55, pp. 811-820, 1991.

6 P. K. Chu, S. Qin, C. Chan, N. W. Cheung and L, A. Larson, "Plasma Immersion Ion Implantation - A Fledgling Technique for Semiconductor Processing”, Mat. Sci. Eng. Reports, 17(6-7), pp. 207-280, 1996.

7 A. Anders (Ed.), Handbook of Plasma Immersion Ion Implantation and Deposition. John Wiley \& Sons, New York, 2000.

8 P. K. Chu, X. Lu, S. S. K. Iyer, and N. W. Cheung, "A New Way to Make SOI Wafers", Solid State Technol., vol. 40, pp. S9-S14, 1997.

9 X. Lu, N. W. Cheung, M. D. Strathman, P. K. Chu, and B. Doyle, "Hydrogen Induced Silicon Surface Layer Cleavage”, Appl. Phys. Lett., vol. 71, pp. 1804-1806, 1997. 
10 X. Lie, M. Samandi, D. Dunne, G. A. Collins, J. Tendys, K. Short, and R. Hutchings, “Cross-sectional transmission electron microscopy characterisation of plasma immersion ion implanted austenitic stainless steel", Surf. Coat. Technol., vol. 85, pp. 28-36, 1996.

11 J. P. Blanchard, "Target temperature prediction for plasma source ion implantation", $J$. Vac. Sci. Technol. B, vol. 12, pp. 910-917, 1994.

12 X. B. Tian, T. K. Kwok, X. C. Zeng, and P. K. Chu, “Target Temperature Simulation during Plasma Immersion Ion Implantation”, 5th IUMRS International Conference on Advanced Materials (IUMRS-ICAM'99), Beijing, China, June 1999.

13 P. K. Chu, S. Qin, C. Chan, N. W. Cheung, and P. K. Ko, "Instrumental and Process Considerations for the Fabrication of Silicon-on-Insulator (SOI) Structures by Plasma Immersion Ion Implantation”, IEEE Trans. Plasma Sci., vol. 26, pp. 79-84, 1998.

14 M. Windner, I. Alexeff, W. D. Jones, and K. E. Lonngren, "Ion Acoustic Wave Excitation and Ion Sheath Evolution”, Phys. Fluids, vol. 13, pp. 2532-2540, 1970.

15 M. Hong and G. A. Emmert, “Two-domensional fluid modeling of time-dependent plasma sheath", J. Vac. Sci. Technol. B, vol. 12, pp. 889-898, 1994.

16 T. E. Sheridan, “Sheath Expansion at a Corner", J. Phys. D, vol. 29, pp. 2725-2728, 1996.

17 M. Chun, B. Kim, J. R. Conrad, R. J. Maty, S. M. Malik, P. Fetherston, and S. Han, "High dose rate effects in silicon by plasma source ion implantation", J. Vac. Sci. Technol. B, vol. 17, pp. 863-866, 1999.

18 X. B. Tian, P. K. Chu, "Direct Temperature Monitoring for Semiconductors in Plasma Immersion Ion Implantation", Rev. Sci. Instrum., vol. 71, pp. 2839-2842, 2000. 


\section{Figure Captions:}

Fig. 1: Calculated ion (heating) flux distributions along the top and side of the sample platen at $20 \mathrm{kV}$ and $40 \mathrm{kV}$ accelerating voltages. Only a quarter of the sample stage (right half of the top and upper half of the side) is shown due to symmetry. Distance from $0 \mathrm{~mm}$ (center of platen) to $76 \mathrm{~mm}$ represents the top surface of the platen, whereas that from $76 \mathrm{~mm}$ to $102 \mathrm{~mm}$ indicates the side of the platen.

Fig. 2: Temperature variation on the top (radial direction) and side (vertical direction) of the sample platen after PIII after: (a) 1 s, (b) 300 s, and (c) 1800 s. Similar to Fig. 1, only a quarter of the sample platen is shown. The upper right corner of the sample platen has the coordinates: radial $=76 \mathrm{~mm}$ and vertical $=26 \mathrm{~mm}$. The conditions are: sample voltage $=-40 \mathrm{kV}$, pulse width $=18 \mu \mathrm{s}$, plasma density $=5 \times 10^{15} \mathrm{~m}^{-3}$, and pulsing frequency $=300 \mathrm{~Hz}$.

Fig. 3: Equilibrium target temperature using a pulsing frequency of $500 \mathrm{~Hz}$ for different implantation voltage, pulse width, and plasma density: (1) voltage $=-40 \mathrm{kV}$, plasma density $=1 \times 10^{16} \mathrm{~m}^{-3}$, pulse width $=12 \mu \mathrm{s}$, (2) voltage $=-40 \mathrm{kV}$, plasma density $=$ $5 \times 10^{15} \mathrm{~m}^{-3}$, pulse width $=18 \mu \mathrm{s},(3)$ voltage $=-20 \mathrm{kV}$, plasma density $=5 \times 10^{15} \mathrm{~m}^{-3}$, pulse width $=12 \mu \mathrm{s}$, and (4) voltage $=-40 \mathrm{kV}$, plasma density $=5 \times 10^{15} \mathrm{~m}^{-3}$, pulse width $=12 \mu \mathrm{s}$.

Fig. 4: Impact of the pulsing frequency on the equilibrium target temperature and external power needed to reach $600^{\circ} \mathrm{C}$ under conditions of voltage $=-40 \mathrm{kV}$, plasma density $=$ $5 \times 10^{15} \mathrm{~m}^{-3}$, pulse width $=18 \mu \mathrm{s}$. The solid symbols represent $\varepsilon=0.1$ whereas the open symbols represent $\varepsilon=0.5$. 


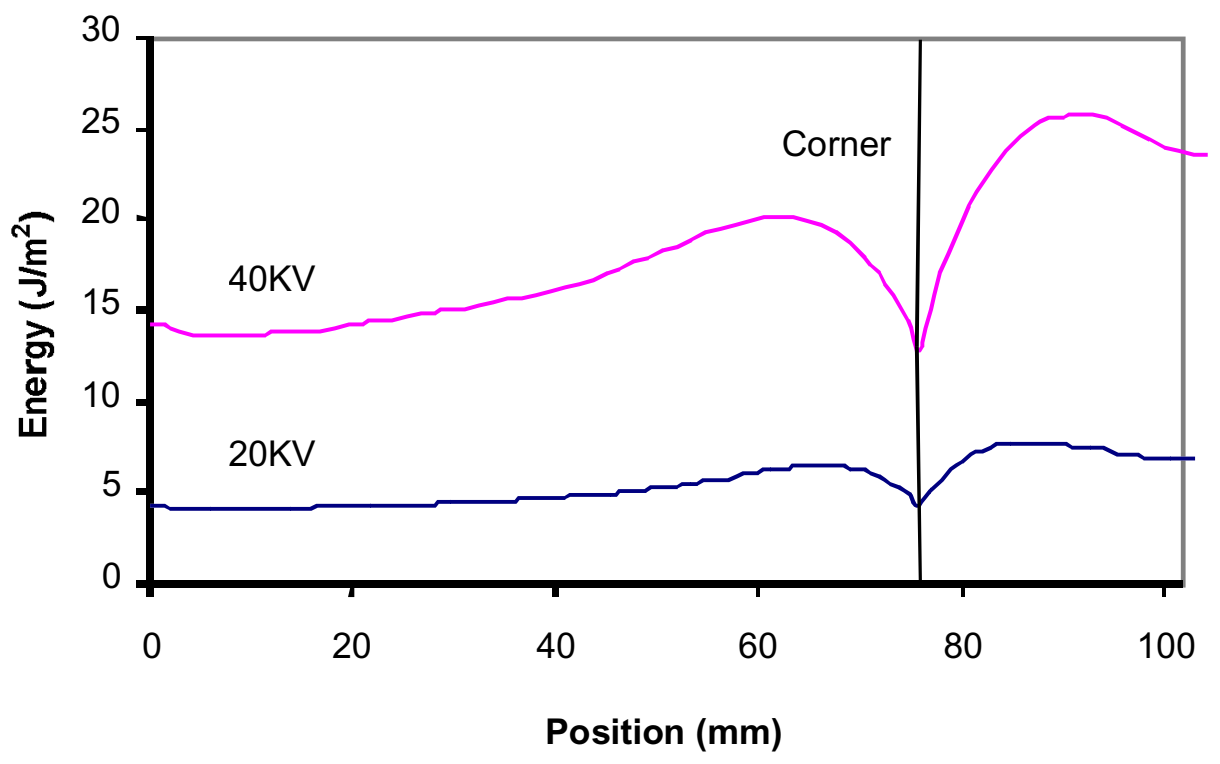

Fig. 1 


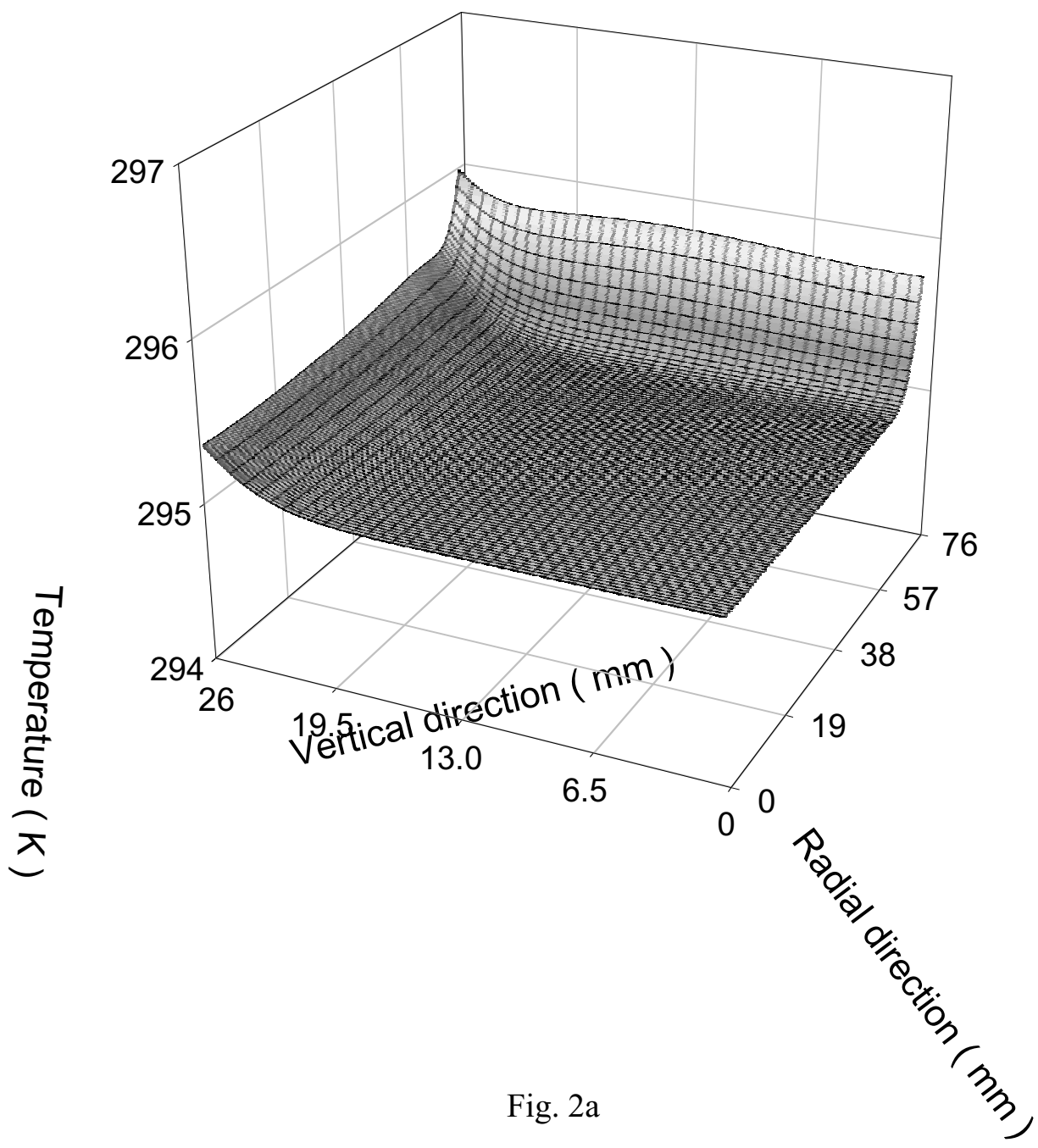




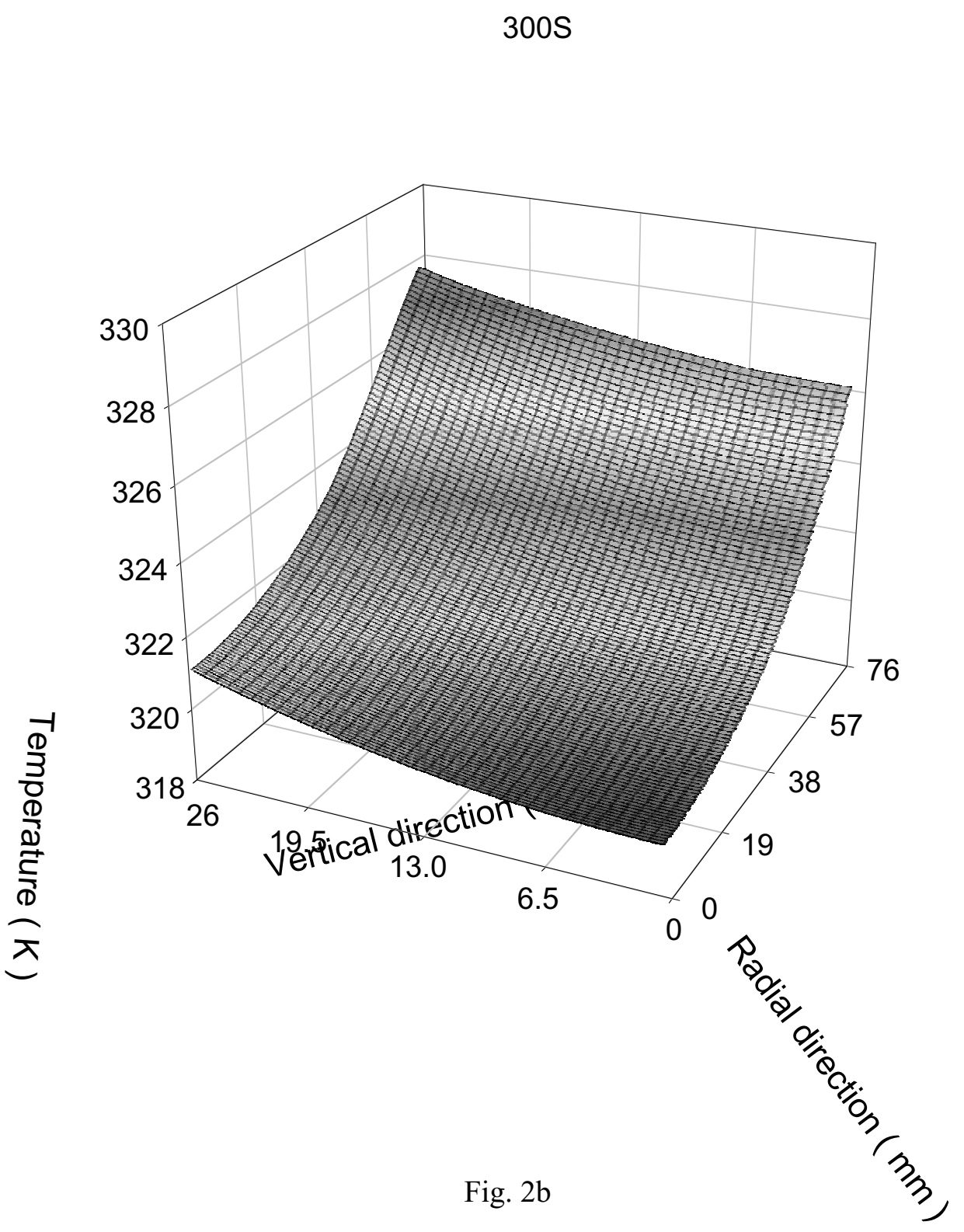


$1800 S$

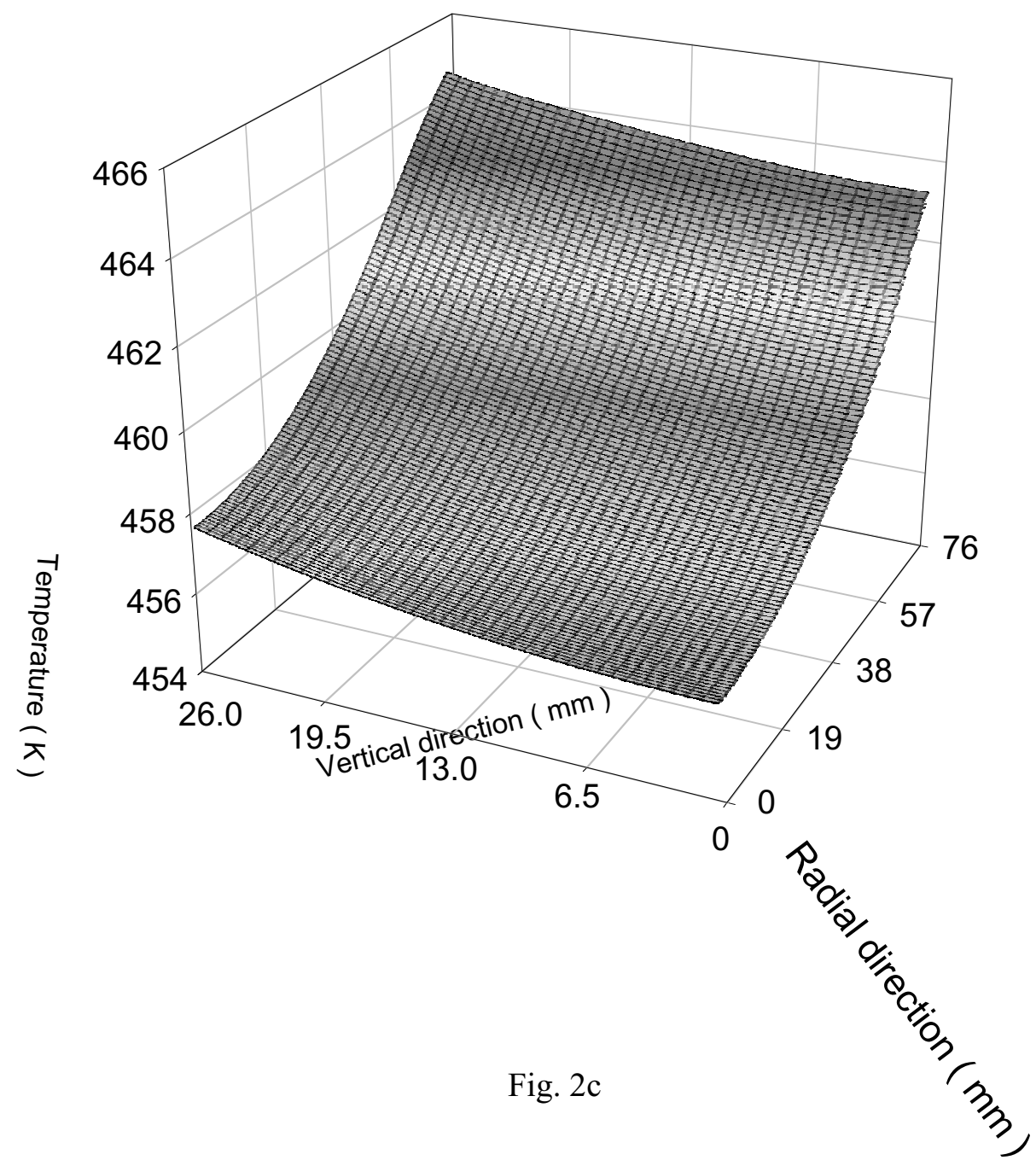




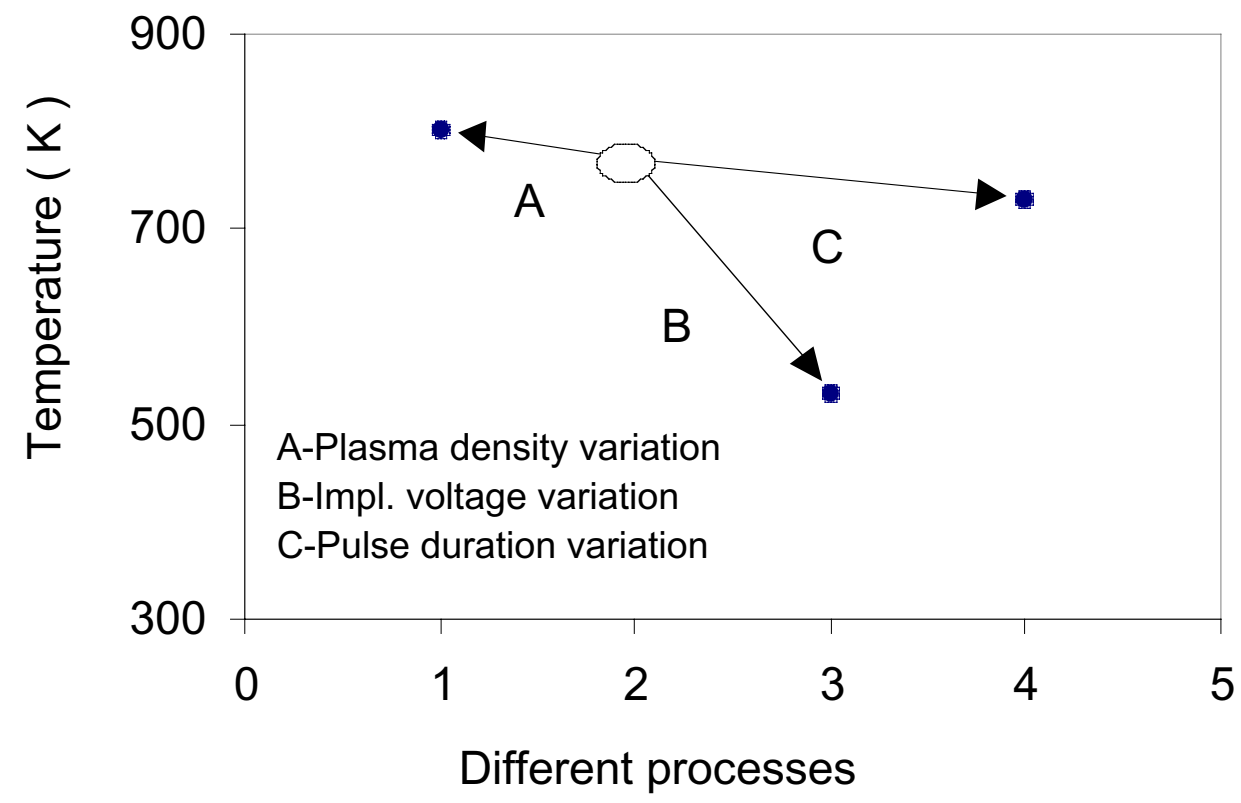

Fig. 3 


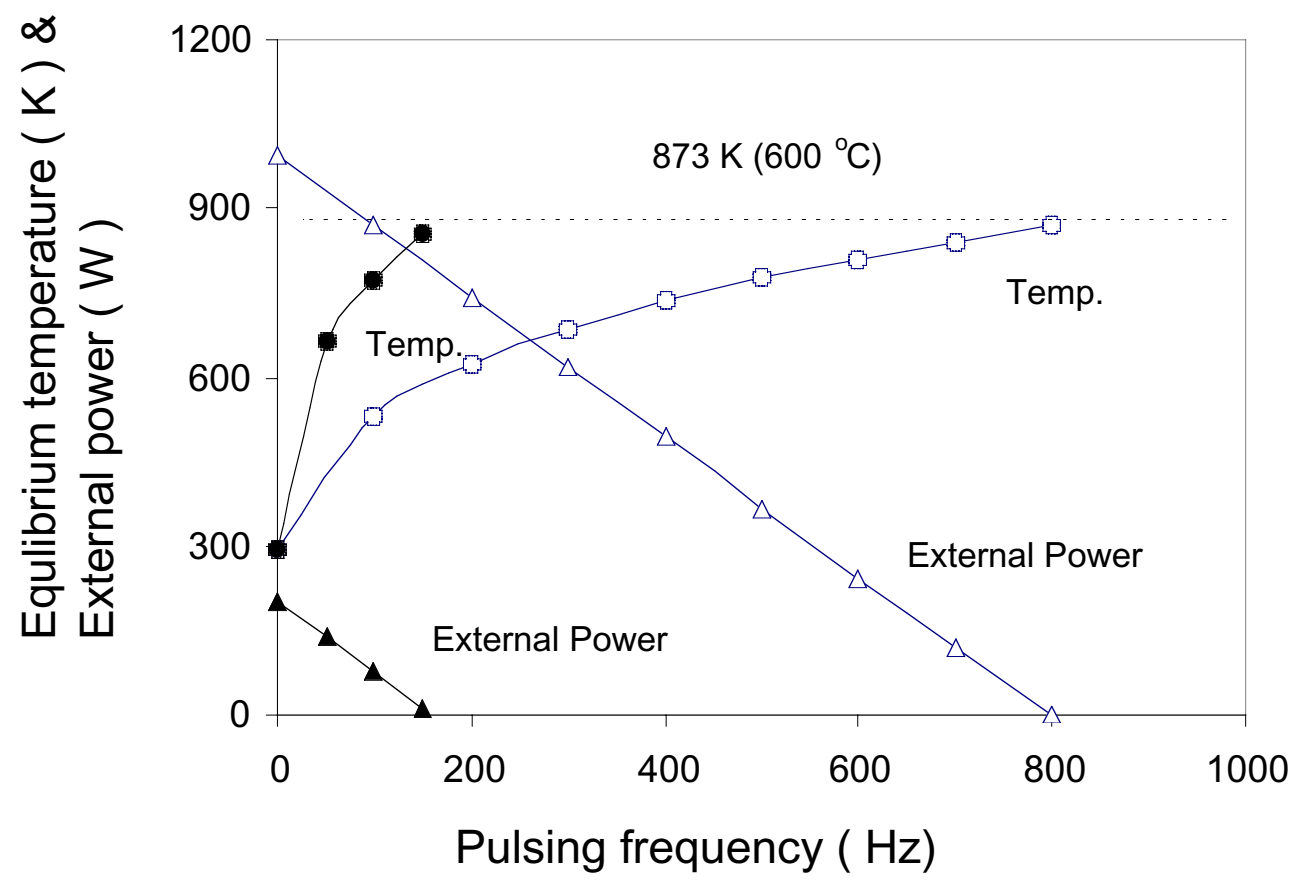

Fig. 4 


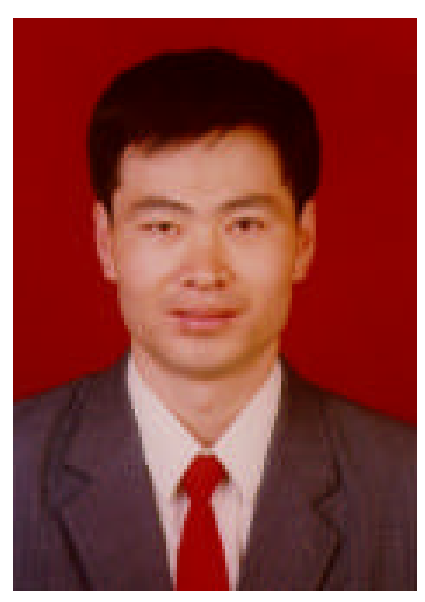

Xiubo Tian (M'01) was born in 1969. He received his BS and MS degrees in materials science and technology from Harbin Institute of Technology (HIT) in China in 1990 and 1993, respectively. He joined the Plasma Laboratory in the City University of Hong Kong in July 1998 as a research staff member and is currently a PhD candidate in the City University of Hong Kong. His research interests are plasma immersion ion implantation, plasma and nitriding equipment and applications, and in-situ monitoring in plasma immersion ion implantation. 


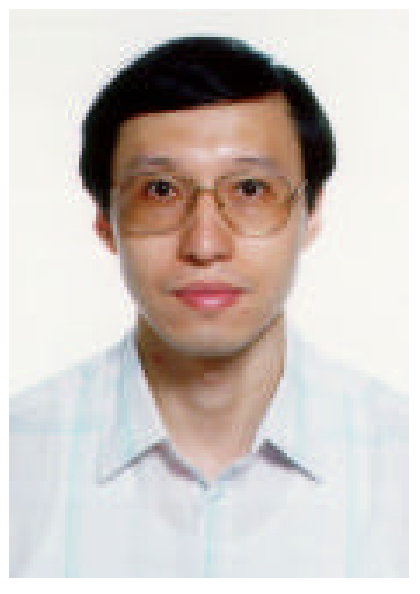

Paul K. Chu (SM'97) received his BS in mathematics from the Ohio State University in 1977 and MS and PhD in chemistry from Cornell in 1979 and 1982, respectively. He joined Charles Evans \& Associates in California in 1982 and started his own business in 1990. He later joined the City University of Hong Kong as a visiting faculty member. He is currently Professor (Chair) of Materials Engineering in the Department of Physics and Materials Science of the City University of Hong Kong. He also holds concurrent professorship at Fudan University, Peking University, Southwest Jiaotong University, and Southwestern Institute of Physics in China. His research activities include plasma processing technology, microelectronics processing, and materials characterization. He is author / co-author of over 400 publications and has 6 US patents. He is Fellow of the Hong Kong Institution of Engineers (HKIE), senior member of IEEE, and an executive member of the International Plasma-Based Ion Implantation Committee. He serves on the Engineering Panel of the Hong Kong Research Grants Council (RGC) and the Editorial Board of Nuclear Instruments and Methods in Physics Research B. 


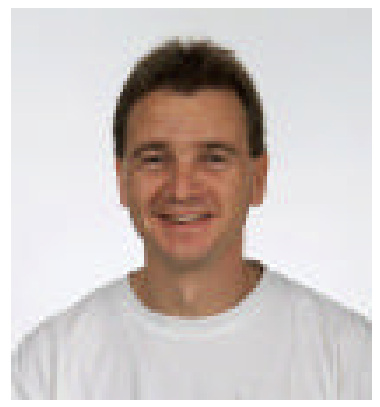

André Anders (M '94, S '98, F '01) is the Group Leader of the Plasma Applications Group at Lawrence Berkeley National Laboratory (LBNL), Berkeley, California. He studied physics in Wroclaw, Poland, Berlin, Germany, and Moscow, Russia. He holds an M.S. degree ('84) and Ph.D. degree ('87) in physics from Humboldt University, Berlin. He was a Staff Scientist at the Academy of Sciences in Berlin (1987-1991) and is affiliated with LBNL since 1992. His research includes vacuum arc plasma and ion sources, gas plasma sources, ion implantation, plasma immersion ion implantation, and thin film synthesis. He is the author of A Formulary for Plasma Physics (Berlin: Akademie-Verlag, 1990) and the editor and coauthor of the Handbook of Plasma Immersion Ion Implantation and Deposition (New York: Wiley, 2000). He has published more than 100 papers in refereed journals. He is the Secretary of the Permanent International Scientific Committee of the International Symposia on Discharges and Electrical Insulation in Vacuum and serves in several other international committees. He received the Chatterton Award (1994) and an R\&D 100 Award (1997). He is a Fellow of IEEE, and a member of MRS, AVS, SVC and IoP (UK). 